\title{
Stenotrophomonas maltophilia Bacteraemia: Analysis of 33 Episodes Occurred in the ICU at the University Hospital in Sousse, Tunisia
}

\author{
Nadia Jaidane1, Cherifa Chaouech1, Walid Naija², Nourddine Boujaafar'1, Olfa Bouallegue ${ }^{1}$ \\ ${ }^{1}$ Emerging Bacterial Resistance and Safety of Care (UR 12 SP 37), Department of Microbiology, University \\ Hospital of Sahloul, Sousse, Tunisia \\ ${ }^{2}$ Department of Anesthesiology and Critical Care Medicine, University Hospital of Sahloul, Sousse, Tunisia \\ Email: ${ }^{*}$ Nadia.jaidane@gmail.com
}

Received 4 July 2014; revised 25 August 2014; accepted 27 September 2014

Copyright (C) 2014 by authors and OALib.

This work is licensed under the Creative Commons Attribution International License (CC BY). http://creativecommons.org/licenses/by/4.0/

(c) (i) Open Access

\section{Abstract}

Background: Stenotrophomonas maltophilia is a multidrug-resistant, Gram negative bacillus that has emerged as an opportunistic pathogen associated with high morbidity and mortality rates. Aim: The aim of this study is to describe the characteristics of patients with bloodstream infections due to $S$. maltophilia. Methods: A descriptive retrospective study was performed at the ICUs over seven-year period in a teaching hospital. Cases of SMB were identified through a review of clinical microbiology laboratory and patient's records were retrieved for analysis. Findings: There were 22 deaths $(71 \%) 15 \pm 12$ days after the bactaeremia. In our study, more than half of patients $(17 / 31)$ was exposed to broad-spectrum antibiotic specifically imipinem (IMP) before their positive culture. Antibiotic susceptibility testing revealed that isolates were most sensitive to ciprofloxacin $(84 \%)$ and to trimethoprim-sulfamethoxazole $(71 \%)$. A probable portal of entry was identified in $\mathbf{2 7 . 3 \%}$ of cases and $\mathbf{5 7 \%}$ were catheter-related. Conclusion: Our results were similar to those described by other authors reported in the literature in the last 20 years. Prevention of $S$. maltophilia acquisition and infection depends on higher emphasis on control of antimicrobial consumption and consideration of environmental reservoirs.

\section{Keywords}

Stenotrophomonas Maltophilia, Bacteraemia, ICU, Risk Factors, Mortality

Subject Areas: Epidemiology, Infectious Diseases

${ }^{*}$ Corresponding author.

How to cite this paper: Jaidane, N., Chaouech, C., Naija, W., Boujaafar, N. and Bouallegue, O. (2014) Stenotrophomonas maltophilia Bacteraemia: Analysis of 33 Episodes Occurred in the ICU at the University Hospital in Sousse, Tunisia. Open Access Library Journal, 1: e954. http://dx.doi.org/10.4236/oalib.1100954 


\section{Introduction}

Stenotrophomonas maltophilia is emerging as an opportunistic nosocomial pathogen which is widespread in the environment [1] [2]. Although not highly virulent, reports indicate that infection with this organism is associated with significant morbidity and mortality particularly in severely compromised patients [3] [4]. Infection with this pathogen commonly manifests as bacteraemia [5] [6], but it also may cause a wide range of other infections [7]-[9]. Progress has been made in identifying risk factors for the acquisition of severe S. maltophilia infections [10]. Thus, clinical manifestations due to this strain was usually related to previous exposure to broad-spectrum antibiotics, prolonged hospitalization, intensive care unit (ICU) stay, mechanical ventilation, the use of intravascular devices and tracheostomy [3] [8] [11]-[16]. Therapy for infections with this pathogen is challenging because of its intrinsic resistance to most antimicrobial agents and the debilitated state of patients [17] [18]. Control of antibiotic use has been identified as a cornerstone of prevention of S. maltophilia infections in hospitals [19] [20], in addition to avoidance of prolonged implantation of foreign devices and reinforcement of hygiene practices [16]. The aim of this study is to describe clinical, microbiological, and epidemiologic features of S. maltophilia bacteraemia (SMB) in the three ICU at the Sahloul Hospital in Sousse, Tunisia

\section{Materials and Methods}

\subsection{Patient Source, Period of Study and Case Finding}

The Sahloul university hospital is a 629-bed teaching hospital with specialty services including operating room and five intensive care units (ICU). A descriptive retrospective study was performed at the ICUs over seven-year period (January 2004-July 2011).

Cases of SMB were identified through review of clinical microbiology laboratory reports. When this organism was cultured from another body site in our patient group it wasn’t recorded. For the Clinical data we've used information from the medical record of each patient. Patients with polymicrobial bacteraemia were included in the study.

An infection was defined nosocomial acquired in an ICU if it occurred more than 2 days after admission.

Patients were considered to have received previous antibiotic treatment if this took place in the week before the development of the bacteraemia, and if it was administered for at least 48 hours.

Considered as Broad spectrum antibiotic (BSA) molecules which are effective against a wide range of microorganisms such as carbapenems.

Antibiotic therapies were considered appropriate if they included at least one drug active in vitro against the isolates from blood culture.

\subsection{Microbiological Data}

The blood culture system was the "BacT ALERT 3D (BIOMERIEUX-FRANCE)".

S. maltophilia isolates were identified in the clinical laboratory by biochemical tests and the Analytical Profile index procedure (API 20-NE-Biomérieux, France) supplemented by oxidase and DNase testing.

\subsection{Antimicrobial Susceptibility Testing}

A standardized disk diffusion method based on MH agar was performed by standardized methods recommended by the Comité de l’ Antibiogramme de la Société Française de Microbiologie (CA-SFM) [21].

The inoculum suspension is prepared by selecting several morphologically similar colonies from overnight growth (16 - $24 \mathrm{~h}$ of incubation) on a non-selective medium with a sterile loop and suspending the colonies in sterile saline $(0.85 \% \mathrm{NaCl} \mathrm{w} / \mathrm{v}$ in water). The density of the suspension is adjusted to McFarland 0.5 standard (1 - 2*108 CFU/ml)

A sterile cotton swab is dipped into the inoculum suspension which is spread evenly over the entire surface of the agar plate by swabbing in three directions. Disks are applied firmly on the agar surface within 15 min of inoculation of the plates. It is important that zone diameters can be reliably measured Within 15 min of application of antimicrobial disks, the plates are inverted and incubated at 35, 1C for 16 - $20 \mathrm{~h}$.

After incubation, inhibition zones are read at the point where no obvious growth is detected by the unaided eye when the plate is held about $30 \mathrm{~cm}$ from the eye. The inhibition zone diameters are measured to the nearest 
millimeter with a ruler. Zone diameters are interpreted and categorized as susceptible, intermediate or resistant according to the CA-SFM clinical breakpoint tables [21].

\subsection{Statistical Analysis}

Descriptive statistics was used for all the studied variables. Satatistical analyses were performed using SPSS 17.0 Statistical package.

\section{Results}

From 2004 to 2011, 33 episodes of SMB were identified in 31 patients. All records were retrieved for analysis. Two patients had two episodes of bacteraemia separated respectively by 10 days and 35 days. Ninty three percent of these episodes were nosocomial acquired at the ICU. Based on microbiology laboratory data the annual frequency of $S$. maltophilia blood isolation remained constant during this period (endemic evolution) except two epidemic periods occurred in 2005 and in 2007, not investigated (Figure 1).

Thirty three episodes were collected via 31 patients. The mean duration of stay prior to SMB was $29 \pm 23$ days. There were 22 deaths in the 31 patients (71\%). The mean time of death after the development of SMB was $15.6 \pm 12$ days (range from 3 to 44 days).

Among cases, 63.6\% developed several nosocomial infections $20 \pm 17$ days prior to ICU-acquired SMB (bactereamia in $45.8 \%$ of the cases, urinary tract infection in $14.6 \%$ of the cases, lung infection in $20.8 \%$ of the cases). All These infections were microbiologically documented and related to strains other than S. maltophilia such us A. baumannii $(25 \%(\mathrm{n}=11))$, E. cloacae, E. coli, K. pneumoniae and other enterobacteriacae $(41 \%(\mathrm{n}=$ 18)).

More than half $51.6 \%(n=16)$ of the patients had at least 1 surgical procedure during their hospitalization. Patient characteristics are presented in Table 1.

In our study, 30.3\% ( $=10)$ of the episodes were polymicrobial and the additional isolates included A. baumannii (70\%) imipinem resistant, K. pneumoniae Extended Spectrum Beta Lactamase (ESBL) and Pseudomonas aeruginosa (P. aeruginosa) Ceftazidime (CFZ) resistant. A probable portal of entry, with isolation from the site preceding SMB was identified in $27.3 \%(\mathrm{n}=9)$ of bacteraemic episodes. This included; CVCs $(55.6 \%(\mathrm{n}=$ 5)), urine and pleural fluid. In our series, $77.4 \%(n=24)$ did not have a clinically apparent portal of entry but $91.7 \%$ of these individuals had a central catheter in place. Data regarding invasive procedures are summarized in Table 2. Nearly $77 \%(n=24)$ of cases had received severel antibiotics prior to bactereamia caused by S. maltophilia, especially imipinem (IMP). Details are shown in Table 3.

Antimicrobial susceptibility data were derived from results of 33 isolates in 31 patients. All isolates were resistant to IMP. Approximately $85.4 \%$ were ciprofloxacin susceptible and $68.3 \%$ were susceptible to Trimethoprim-sulfamethoxazole (SXT). Resistances of S. maltophilia to CFZ and Fosfomycin (FOS) were 56\% and 81.3\% respectively. The antimicrobial susceptibility of blood isolates is shown in Figure 2.

Definitive antimicrobial treatment was possible in the 33 episodes of SMB, 19 (57.6\%) were treated with antibiotic therapy included an appropriate agent in the most cases (89.5\%). A monotherapy was administrated in 8 cases (42.1\%) and a biotherapy in 7 cases (36.8\%). CIP were the most prescript (31.6\%).

\section{Discussion}

The objective of our study is to describe clinical, microbiological, and epidemiologic features of SMB. Although,

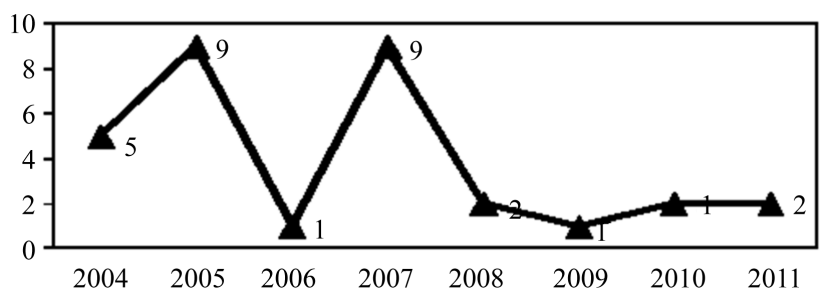

Figure 1. Distribution of $S$. maltophilia positive blood culture over seven years. 
Table 1. Underlying characteristics of patients with bacteraemic episodes.

\begin{tabular}{|c|c|}
\hline Characteristics $n=31\left({ }^{*} n=33\right)$ & \\
\hline Mean age mean \pm SD [years] (range) & $54 \pm 16(19-87)$ \\
\hline Gender & \\
\hline - Male & $64.5 \%(20)$ \\
\hline - Female & $35.5 \%(11)$ \\
\hline Cause of admission to ICU & \\
\hline - Trauma & $29 \%(9)$ \\
\hline - Medical & $45.2 \%(14)$ \\
\hline - Urgent Surgery/Programmed surgery & $25.8 \%(8)$ \\
\hline Duration of stay in the ICU prior to SMB & $29 \pm 23(5-108)$ \\
\hline Past medical history & $64.5 \%(20)$ \\
\hline - Diabetes & $55 \%(11)$ \\
\hline$\checkmark \quad$ type 1 diabetes mellitus (ID) & $45.5 \%(5)$ \\
\hline$\checkmark \quad$ type 2 diabetes mellitus (NID) & $54.5 \%(6)$ \\
\hline - Hypertension & $65 \%(13)$ \\
\hline - Cancer & $25 \%(5)$ \\
\hline - Chemotherapy/ Radiotherapy & $10 \%(2)$ \\
\hline Other nosocomial infections before the bacteriemic episodes with S. maltophilia* & $63.6 \%(21)$ \\
\hline $\begin{array}{ll}\text { Coexisting conditions } \\
\text { - } & \text { Major surgery } \\
\text { - } & \text { Purulent sputum } \\
\text { - } & \text { Bronchopneumopathy } \\
\text { - } & \text { Septic shock } \\
\text { - } & \text { Visceral damage } \\
\text { - } & \text { Respiratory failure } \\
\text { - } & \text { Kidney failure }\end{array}$ & $\begin{array}{l}51.6 \%(\mathrm{n}=16) \\
51.6 \%(16) \\
45.2 \%(14) \\
12.9 \%(4) \\
12.9 \%(4) \\
12.9 \%(4) \\
9.7 \%(3) \\
9.7 \%(3)\end{array}$ \\
\hline Mechanical ventilation & $74.2 \%(n=23)$ \\
\hline Central venous catheter & $93.5 \%(n=29)$ \\
\hline Prior antibiotics therapy & $77 \%(n=24)$ \\
\hline Surgical procedure & $51.6 \%(\mathrm{n}=16)$ \\
\hline Prior use of IMP & $70.8 \%(17)$ \\
\hline Polymicrobial bacteraemia & $30.3 \%(n=10)$ \\
\hline Death & $71 \%(22)$ \\
\hline
\end{tabular}

Table 2. Invasive procedure.

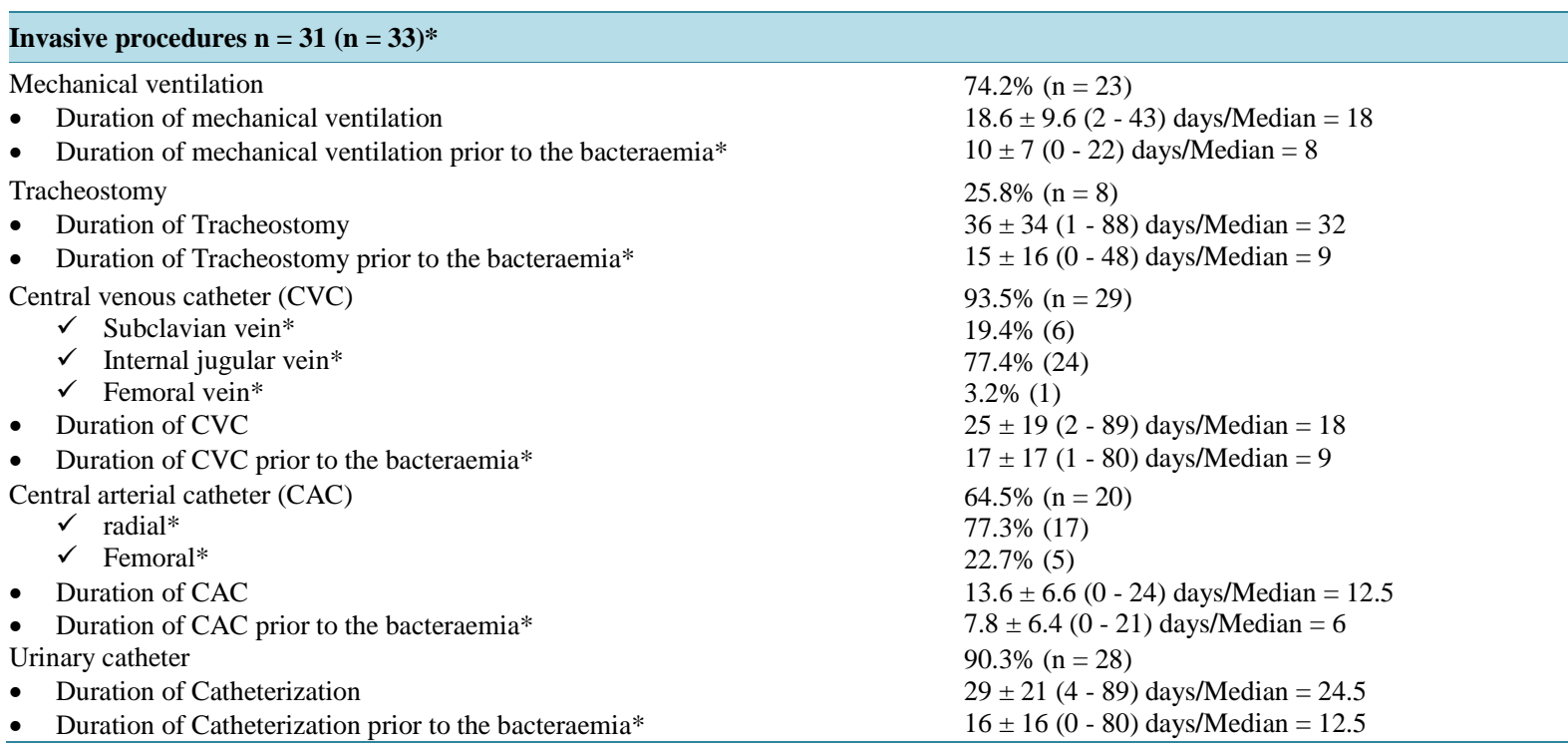


Table 3. Exposure to antimicrobial agent $(n=33)$.

\begin{tabular}{|c|c|c|c|}
\hline Antibiotic & Cases \% (no.) & Duration (days) & $\begin{array}{c}\text { Duration of administration prior to } \\
\text { the bacteraemia (days) }\end{array}$ \\
\hline Amoxicillin-clavulanic acide (AMC) & $45.8(11)$ & $4.4 \pm 2.65(2-10)$ & $20.9 \pm 22.62(3-80)$ \\
\hline Cefotaxim (CTX) & $20.8(5)$ & $7.2 \pm 2.95(4-10)$ & $8 \pm 6.7(1-19)$ \\
\hline Imipenem (IMP) & $70.8(17)$ & $22.9 \pm 8.44(8-40)$ & $15.3 \pm 13.93(3-58)$ \\
\hline Gentamicin (GM) & $83.7(20)$ & $5.75 \pm 3.37(1-20)$ & $15.55 \pm 13.85(1-65)$ \\
\hline Ciprofloxacin (CIP) & $16.7(4)$ & $10.5 \pm 4.43(6-16)$ & $13.3 \pm 14.2(0-31)$ \\
\hline Vancomycin (VA) & $45.8(11)$ & $18.45 \pm 5.78(7-30)$ & $13.45 \pm 12.9(0-65)$ \\
\hline Colistine (CS) & $12.5(3)$ & $18.3 \pm 7.76(12-27)$ & $12.2 \pm 9.4(3-25)$ \\
\hline Rifampicin (RA) & $20.8(5)$ & $12.6 \pm 8.84(3-27)$ & $9.2 \pm 8.4(1-24)$ \\
\hline
\end{tabular}

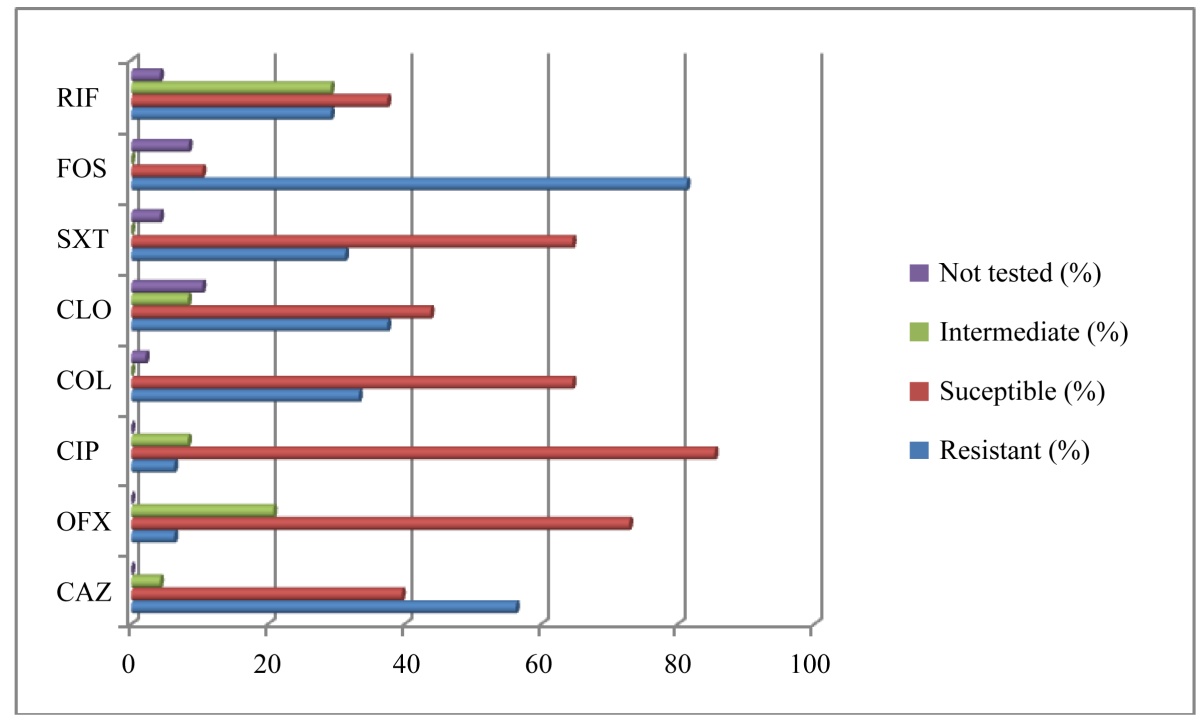

Figure 2. Antibiotic susceptibility of S. maltophilia for 33episodes of SMB Rifampicin (RIF), Fosfomycin (FOS), Trimethoprim-sulfamethoxazole (SXT); chloramphenicol (CLO); Colistine (COL) ciprofloxacin; (Cip); Ofloxacin (OFX), ceftazidime (CFZ)).

this study was restricted to the ICUs setting and had several limitations. First, the absence of a control group is a limitation recognised by the authors, and has previously been a valuable inclusion in studies of this kind [22] [23]. Second, we've included only 31 cases, indeed most previous studies had analysed a small population and have been mostly retrospective and only few of these included more than 35 patients [5] [11] [22] [24]. Finally, the heterogeneity of patients, lack of appropriate statistical analysis and lack of definition of nosocomial episodes of bacteraemia, lack of the criteria for attributing death to the bacteraemic episode were not specified, thus we had difficulties of determining a precise cause of death for our population who had multiple active co-morbidities. The characteristics in our population and those described by other authors are shown in Table 4 .

Risk factors for acquiring S. maltophilia infection especially in the ICU are associated with a severely compromised health status and underlying disease, a long-term hospitalization or ICU stay, medical treatment involving indwelling devices such as intravascular catheters and exposure to broad-spectrum antimicrobials [1] [2] [13].

Usually S. maltophilia primarily affects patients with co-morbid illness such as immunosuppression, cystic fibrosis, malignancies, neutropenia, corticotherapy, chronic disease and severe trauma [3] [31]. Comorbid medical conditions in our patients were dissimilar to other studies [4] [17] [31] [33]. Most of comorbidities cited previously were uncommon in our population. Third of our patients 29\% (9) had major trauma among them 6 were died $(66.7 \%)$ and $64.5 \%(n=20)$ of patients had chronic disease. In this group 15/20 (75\%) were died versus $7 / 11(63.6 \%)$ in the other group. The difference was not statistically significant $(P=0.7)$ by chi-square 
Table 4. Baseline characteristics of the patients in comparison with selected previously reported studies.

\begin{tabular}{lccccccc}
\hline \multicolumn{1}{c}{ Authors } & $\begin{array}{c}\text { No. of cases } \\
\text { /episodes }\end{array}$ & Age & $\begin{array}{c}\text { Duration of } \\
\text { hospitalization } \\
\text { (days) }\end{array}$ & $\begin{array}{c}\text { Previous } \\
\text { Imipinem }\end{array}$ & Intubation & CVC & Death \\
\hline Our study & 33 & $54 \pm 16$ & $19 \pm 17$ & $71 \%(17)$ & $74 \%(\mathrm{n}=23)$ & $93 \%(\mathrm{n}=29)$ & $71 \%$ \\
Lai et al. (2004) [1] & 84 & $62 \pm 2$ & $24(3-100)$ & $42 \%$ & $36 \%$ & $83 \%$ & $33 \%$ \\
Senol et al. (2002) [2] & 30 & 46 & $11 \pm 18$ & $33 \%(\mathrm{n}=10)$ & $13 \%$ & $80 \%(\mathrm{n}=24)$ & $27 \%$ \\
Friedman et al. (2002) [5] & 45 & 54 & 19 & $40 \%(18)$ & $48 \%(21)$ & $84 \%(38)$ & $18 \%(\mathrm{n}=8)$ \\
Apisarnthanarak 2003 [6] & 13 & $\mathrm{ND}$ & 14 & $54 \%(\mathrm{n}=7)$ & $\mathrm{ND}$ & $61 \%(\mathrm{n}=8)$ & $\mathrm{ND}$ \\
Muder et al. (1996) [11] & 91 & $\mathrm{ND}$ & $\mathrm{ND}$ & $\mathrm{ND}$ & $20 \%$ & $82 \%$ & $21 \%$ \\
Araoka H et al. (2010) [18] & 53 & 57 & $\mathrm{ND}$ & $31 \%$ & $\mathrm{ND}$ & $57 \%(30)$ & $51 \%$ \\
Micozzi et al. (2000) [22] & 37 & 29 & 29 & $\mathrm{ND}$ & $\mathrm{ND}$ & $86 \%(32)$ & $24 \%$ \\
Victor et al. 1994 [23] & 14 & $34(7-68)$ & 23 & $50 \%$ & $\mathrm{ND}$ & $100 \%$ & $92 \%$ \\
Elting et al. 1990 [24] & 60 & 51 & 13 & $50 \%$ & $\mathrm{ND}$ & $88 \%$ & $38 \%$ \\
Wang et al. (2004) [25] & 50 & $28-94$ & 36 & $56 \%$ & $40 \%$ & $60 \%$ & $62 \%$ \\
Cheong et al. (2008) [26] & 109 & $53 \pm 15$ & $>30$. & $\mathrm{ND}$ & $16 \%$ & $76 \%(\mathrm{n}=83)$ & $\mathrm{ND}$ \\
Nseir 2006 [27] & 38 & $63 \pm 12$ & $14 \pm 11$ & $15 \%(\mathrm{n}=6)$ & $84 \%$ & $92 \%(35)$ & $60 \%$ \\
Metan G et al. 2005 [28] & 41 & 51 & 24 & $75 \%$ & $27 \%$ & $90.2 \%$ & $4 \%$ \\
Ubeda P et al. 1998 [29] & 26 & 40 & $\mathrm{ND}$ & $25 \%$ & $50 \%$ & $73 \%(\mathrm{n}=19)$ & $20 \%$ \\
Villarino et al. 1992 [30] & 45 & 46 & 23 & $38 \%(\mathrm{n}=17)$ & $84 \%$ & $\mathrm{ND}$ & $33 \%(\mathrm{n}=15)$ \\
Ansari S. et al. 2007 [31] & 54 & 55 & 16 & $46 \%$ & $\mathrm{ND}$ & $\mathrm{ND}$ & $22 \%$ \\
Gopalakrishnan. 1999 [32] & 143 & 60 & 13 & $7 \%$ & $82 \% \%$ & $\mathrm{ND}$ & $47 \%$ \\
\hline & & & & & &
\end{tabular}

test. A higher incidence of diabetes was demonstrated in our study when compared with other reported series [2] [24] [32].

A number of studies have pointed to an association between ICU stays and the subsequent increasing of incidence, mortality and morbidity due to S. maltophilia [11] [25] [31].

The mean duration of stay in our study prior to SMB was $19 \pm 17$ similar to other reports [1] [5] [23] [28] [30] [31] [34], who reported a duration ranged between 16 and 23 days. Whereas some authors found more extended duration of stay ( $>29$ days) [22] [25] [26]. Others [2] [6] [24] [27] [32], have reported a less extended duration ranged between 11 and 14 days. In addition, in a case control study dealing with 53 cancer patients by Ansari et al. [31], ICU stay within 30 days before the isolation of S. maltophilia was found to be significantly associated with the emergence of Multi-Drug Resistant (MDR) S. maltophilia and had the highest OR of all the risk factors. Micozzi et al. [22] included in his study a control group of patients with bacteraemia due to $P$. aeruginosa. He observed that more cases of SMB are developed during hospitalization but he didn't find any statistical significant difference between a control group and his population with SMB. In addition, Metan et al. [28] had found that the patients who died within 30 days after the onset of SMB had a higher rate of intensive care unit stay. The importance of intravascular devices exposure in the pathogenesis of SMB is, however, becoming increasingly recognized such as mechanical ventilation, tracheostomy [4] [11] [12] [23] [24].

The majority of patients in our study have had indwelling intravascular devices related to the fact that our cases was exclusively collected from ICU and patients had characteristics that indicated significantly worse underlying severity of illness. The presence of an indwelling CVC has been previously reported for $78 \%$ - $86 \%$ of patients [23] [24] [35] [36]. This range is lower than the proportion in our figure which was 93.5\%. Wang [25] in his univariate analysis revealed that CVC and mechanical ventilation were significantly associated with a high crude mortality rate. This also tallied with several other studies who implicated CVC [17] [30] and mechanical ventilation [11] [17] [23] [24].

The portal of entry of $S$. maltophilia infection is frequently unknown. Our results highlight the fact that Central-venous lines are the most common source of SMB (SMB was attributed to a CVC infection in almost 55.6\% of our patients). This finding is consistent with those of other reports [5] [10] [11] [22] [33] [37] [38]. 
In cases where there is no obvious source of infection it has been suggested that these devices may be the primary source [16]. Muder et al. [11] reported that in their series 56\% of cases with SMB did not have a clinically apparent portal of entry but $84 \%$ of these individuals had a CVC in place. Senol [2] found that 30\% of cases had no apparent primary source of infection, but all had CVCs in place. In our study $77.4 \%$ didn't have a clinically apparent portal of entry. As $91.7 \%$ of these patients had a CVC in place, it is likely that the catheter was the ultimate source of infection in many of these patients.

Most studies on risk factors for the emergence of S. maltophilia have revealed that Selective pressure caused by the overuse of broad-spectrum antibiotics, especially carbapenems, predispose to infection or colonization with S. maltophilia [13] [31] [32]. In the present study, we found a high frequency in IMP prescription prior to SMB, which is consistent with previous reports, in which between $25 \%$ and $43 \%$ of patients received this antibiotic [5] [11] [23] [35]. The use of carbapenems has also been established as the primary risk factor for S. maltophilia infections [31] [39]. Prior therapy with IMP was 10 times more frequent amongst cases of S. maltophilia than in matched controls in a previous case-control study conducted at the University of Texas [12]. Many other studies, used multi-variate analysis, found carbapenem use before admission to the ICU to be an independent risk factor [12] [27] [30] [40] [41].

An association between the emergence of this strain and the broad-spectrum antibiotics other than IMP, such as aminoglycosides, fluoroquinolones, and extended-spectrum cephalosporins, has been previously documented [2] [15] [42]. Meyer [40], in his univariate analysis, added strength to this findings by demonstrating a significant correlation between carbapenems, other broad-spectrum antibiotics and total antibiotic consumption with the incidence density of $S$. maltophilia.

Susceptibility study among the strains isolated from the 33 episodes of SMB showed that the most effective antibiotics in vitro were CIP (85.4\%), SXT (68.3\%), C (43.9\%) and CFZ (43.9\%). These observations coupled with other reports from different institution [1] [2] [11] [5] [12] [42]-[45] (Table 5).

The major problem which challenges the laboratorians and clinicians is the emergence of resistance to SXT. Indeed, SXT remains the most effective drug for the treatment of $S$. maltophilia infections despite the view that this drug is only bacteriostatic for most isolates [11] [7] [23] [16] [43] [46]-[49].

Nevertheless, the emergence of resistance to this agent [42] [47] [50], and allergic reactions or more accurately, hypersensitivity reactions, which are not uncommon, leads to further limitation of available treatment options [16] [47].

We've noted in our study $31.7 \%$ of resistance and this value is alarming. Several reports have shown that the prevalence of strains that are resistant to SXT is increasing and a widely variation in resistance to SXT was documented [22] [16] [51] [49] [52]. Indeed, in Spain, Canada and Latin America, authors reported that 2\% of isolates of S. maltophilia were resistant to SXT [53] [54] [49], 10\% in Europe [49]. Whereas an Italian study reported the highest levels of resistance reached $80.9 \%$ of the isolates [55].

Table 5. Antibiotic susceptibility characteristics of our population in comparison with selected previously reported studies (n $=33)$.

\begin{tabular}{|c|c|c|c|c|}
\hline & Ceftazidime (CFZ) & Ciprofloxacine (CIP) & Sulfamethoxazole (SXT) & Chloramphenicol (C) \\
\hline Our study & $44 \%$ & $85 \%$ & $68 \%$ & $44 \%$ \\
\hline Lai et al. (2004) [1] & $60 \%$ & $83 \%$ & $76 \%$ & $71 \%$ \\
\hline Looney W. et al. (2009) [3] & $5 \%-53 \%$ & $0 \%-82 \%$ & $0 \%-100 \%$ & $1 \%-80 \%$ \\
\hline Morrison et al. (1986) [4] & $4 \%$ & ND & $100 \%$ & ND \\
\hline Friedmann et al. (2002) [5] & $65 \%$ & $60 \%$ & $80 \%$ & $76 \%$ \\
\hline Muder et al. (1996) [11] & $56 \%$ & $62 \%$ & $91 \%$ & ND \\
\hline Micozzi et al. (2000) [22] & $27 \%$ & $19 \%$ & $42 \%$ & ND \\
\hline Wang et al. (2004) [25] & $44 \%$ & $36 \%$ & $60 \%$ & $42 \%$ \\
\hline Ubeda P. et al. (1998) [29] & $62 \%$ & $60 \%$ & $90 \%$ & ND \\
\hline Tsai et al. (2006) [34] & $62 \%$ & $43 \%$ & $14 \%$ & ND \\
\hline Betriu et al. (2001) [43] & $50 \%$ & $15 \%$ & $95 \%$ & $33 \%$ \\
\hline Safdar et al. (2007) [44] & $15 \%-24 \%$ & $16 \%-61 \%$ & $75 \%-98 \%$ & $0 \%$ \\
\hline
\end{tabular}


Betriu [43] showed that resistance to SXT decreased significantly from 16.8\% in 1995-1996 to 4.7\% in 2001 $(P<0.005)$. He also observed an increasing trend in the rates of resistance to ciprofloxacin $(P<0.05)$ from $54 \%$ in $1993-1994$ to $68.7 \%$ in 2001 . These patterns of susceptibility reflected the development the antibiotic prophylaxis practices in the hospitals according to some authors [1] [42] [56].

It is clear that infections caused by S. maltophilia are particularly difficult to manage and should be approached with caution because of many features of resistance of clinical isolates. The concept of "appropriate" therapy for S. maltophilia is challenging. There is ongoing debate, regarding the optimal treatment of $S$. maltophilia infection, about the use of monotherapy versus combination therapy [16] [11] [25] [57] [42] [47]. In our study, among patients $57.6 \%$ were treated with an appropriate agent. Several studies have reported a significant association between survival and administration of appropriate antimicrobial therapy [22] [23] [11] [35]. We did not find such association, most likely because therapy was not controlled in our study. Interest in antibiotic combinations has led to numerous studies of in vitro synergy [30] [5] [43] [58]. The potential benefits of combination antibiotic therapy are well recognised. They include a broadened and more reliable spectrum of activity, enhanced antibacterial activity due to any synergistic or additive effect allowing lower doses of toxic agents to be given, and prevention of the emergence of resistance [3] [11] [46].

About the management of Infection by other means than antimicrobial agents, several investigators have stressed the importance of removing infected CVC [1] [24] [18] [38] [45] [59]. Boktour et al. [33], found that prompt removal of the catheter in cancer patients, in whom Catheter-related SMB was occurred, was found to be associated with a better prognosis. However, Muder and al. [11] reported the successful management of CVCrelated bacteraemia without removal of the device and concluded that there was no standardized protocol for catheter removal and culture.

In our series there was a crude mortality percentage $71 \%$ of $S$. maltophilia bactereamia suggesting that SMB itself serves as a marker of serious pathology. This is higher than reported mortality rates in earlier studies, which ranged from 22\% to 69\% [2] [23] [11] [25] [26] [30] [32] [34]-[36]. Direct attributable mortality is weak, and seems to depend on the severity of the underlying disease. There have been some studies on mortality of $S$. maltophilia bactereamia, but most studies included only small numbers of patients [5] [22]. Furthermore, data regarding risk factors for mortality of S. maltophilia bactereamia are limited and the criteria for attributing death to the bacteremic episode were not specified [26].

Polymicrobial SMB is a frequent occurrence and there was a significant debate about the association of this common characteristic with the mortality rate [24]. Indeed, according to some investigators, the acute mortality rate associated with these polymicrobial bacteraemias is not significantly different from that associated with SMB alone [12] [53]. In our study Polymicrobial bacteraemia occurred in 10 of the 33 cases and the mortality rate was as high as $60 \%$ versus $78.3 \%$ in monomicrobial cases $(P=0.4)$. These percentages were similar to previous studies [1] [23] [11] [35] [6] [10] [18] [25] [29]. The main additional isolate was $A$. baumannii (70\%, $\mathrm{n}=$ 7) similar to prior report from Lai [1], who explained that the increased isolation of Acinectobacter spp. may have been due to antibiotic selective pressure (Table 6).

Table 6. Polymicrobial characteristics of our population in comparison with selected previously reported studies $(n=33)$.

\begin{tabular}{|c|c|c|}
\hline & $\begin{array}{l}\text { Frequency of } \\
\text { polymicrobial } \\
\text { episodes }\end{array}$ & Additional isolates \\
\hline Our study & $30 \%(n=10)$ & A baumannii $(\mathrm{n}=7)$, K. pneumoniae $(n=1), P$. aeruginosa $(n=1)$, Alcaligenes spp. $(n=1)$ \\
\hline Lai et al. (2004) [1] & $27 \%(n=23)$ & Enterobacteriaceae $(\mathrm{n}=10)$, Acinetobacter spp. $(\mathrm{n}=6)$, SARM $(\mathrm{n}=6)$, Enterococcus spp. \\
\hline Morrison et al. (1986) [4] & $64 \%(n=63)$ & $\begin{array}{l}\text { Enterobacter }(\mathrm{n}=14), \text { P. aeruginosa }(n=12), \text { S. aureus }(n=10), \text { E. coli }(n=8) \text {, } \\
\text { K. pneumoniae }(n=7) \text {, Candida }(\mathrm{n}=7) \text {, A baumannii }(\mathrm{n}=4)\end{array}$ \\
\hline Muder et al. (1996) [11] & $40 \%(n=36)$ & $\begin{array}{l}\text { Coagulase-negative staphylococci }(\text { CoNS })(\mathrm{n}=10 \text { isolates }) \text {, other Non fermentative } \\
\text { Gram-negative bacilli (NF-GNB) }(\mathrm{n}=10) \text {, Enterobacteriaceae }(\mathrm{n}=9) \text {, Enterococcus spp. }(7) \text {, } \\
\text { Candida spp }(\mathrm{n}=3)\end{array}$ \\
\hline Araoka et al. (2010) [18] & $38 \%(20 / 53)$ & $\begin{array}{l}\text { Enterococcus spp. }(\mathrm{n}=13), \text { Staphylococcus epidermidis }(\mathrm{n}=5), P . \text { aeruginosa }(\mathrm{n}=4), \text { E. } \\
\text { cloacae }(\mathrm{n}=3) \text {, Methicillin-resistant Staphylococcus aureus }(\mathrm{MRSA})(\mathrm{n}=3) \text {, Enterobacter } \\
\text { aerogenes }(\mathrm{n}=1), \text { Burkholderia cepacia }(\mathrm{n}=1), \text { K. pneumoniae }(\mathrm{n}=1), \text { Bacteroides fragilis } \\
(\mathrm{n}=1)\end{array}$ \\
\hline Micozzi et al. (2000) [22] & $16 \%(6 / 37)$ & Cocci Gram positif \\
\hline Wang et al. (2004) [25] & $16 \%(8 / 50)$ & A. baumannii $(\mathrm{n}=3)$ Enterobacter aerogenes $(\mathrm{n}=2)$ E. coli $(\mathrm{n}=2)$, Candida albicans $(\mathrm{n}=1)$ \\
\hline
\end{tabular}




\section{Conclusion}

Data in our study and the current status of knowledge show that SMB in ICU is a cause of concern. This problematic opportunist is closely related to debilitated host, long-term stay in ICU, indwelling devices and prior exposure to broad-spectrum antibiotics. Therapy for this infection is increasingly challenging for the high level of intrinsic resistance, uncertainties about the value of in-vitro susceptibility testing, and the emergence of resistance to antimicrobials such as SXT and TCC, which are recommended for empirical treatment. Effective control strategies are needed to prevent the spread of this micro-organism. These strategies must emphasize the importance of continued local surveillance of $S$. maltophilia isolation, in addition, the implantation of a multidisciplinary, education-based, antibiotic-resistance management approach and reinforcement of hygiene practices.

\section{Acknowledgements}

The authors would like to thank Pr. Rchid SAID Chief of the ICU and his staff especially Mrs Leila JEGHAM and Mrs Nadia HAFSI, Pr Houssem HMOUDA Chief of the medical intensive care unit for their precious help in acquisition of data and Pr Lamine DHIDEH Chief of the hospital hygiene service for his critical revision of the manuscript.

\section{Conflicts of Interest}

We declare that we have no conflicts of interest.

\section{References}

[1] Lai, C.H., Chi, C.Y., Chen, H.P., et al. (2004) Clinical Characteristics and Prognostic Factors of Patients with Stenotrophomonas maltophilia Bacteremia. Journal of Microbiology, Immunology and Infection, 37, 350-358.

[2] Senol, E., DesJardin, J., Stark, P.C., Barefoot, L. and Snydman, D.R. (2002) Attributable Mortality of Stenotrophomonas maltophilia Bacteremia. Clinical Infectious Diseases, 34, 1653-1656. http://dx.doi.org/10.1086/340707

[3] Looney, W.J., Narita, M. and Mühlemann, K. (2009) Stenotrophomonas maltophilia: An Emerging Opportunist Human Pathogen. The Lancet Infectious Diseases, 9, 312-323. http://dx.doi.org/10.1016/S1473-3099(09)70083-0

[4] Morrison, A.J., Hoffmann, K.K. and Wenzel, R.P. (1986) Associated Mortality and Clinical Characteristics of Nosocomial Pseudomonas maltophilia in a University Hospital. Journal of Clinical Microbiology, 24, 52-55.

[5] Friedman, N.D., Korman, T.M., Fairley, C.K., Franklin, J.C. and Spelman, D.W. (2002) Bacteremia Due to Stenotrophomonas maltophilia: An Analysis of 45 Episodes. Journal of Infection, 45, 47-53. http://dx.doi.org/10.1053/jinf.2002.0978

[6] Apisarnthanarak, A., Mayfield, J.L., Garison, T., et al. (2003) Risk Factors for Stenotrophomonas maltophilia Bacteremia in Oncology Patients: A Case-Control Study. Infection Control and Hospital Epidemiology, 24, 269-274. http://dx.doi.org/10.1086/502197

[7] Schaumann, R., Stein, K., Eckhardt, C., Ackermann, G. and Rodloff, A.C. (2001) Infections Caused by Stenotrophomonas maltophilia-A Prospective Study. Infection, 29, 205-208. http://dx.doi.org/10.1007/s15010-001-1055-4

[8] Hanes, S.D., Demirkan, K., Tolley, E., et al. (2002) Risk Factors for Late-Onset Nosocomial Pneumonia Caused by Stenotrophomonas maltophilia in Critically Ill Trauma Patients. Clinical Infectious Diseases, 35, 228-235. http://dx.doi.org/10.1086/341022

[9] Khan, I.A. and Mehta, N.J. (2002) Stenotrophomonas maltophilia Endocarditis: A Systematic Review. Angiology, 53, 49-55.

[10] Tunger, O., Vural, S., Cetin, C.B., Keles, G., Borand, H. and Gazi, H. (2007) Clinical Aspects and Risk Factors of Nosocomial Stenotrophomonas maltophilia Bacteremia Episodes in a Turkish Intensive Care Unit. Journal of Chemotherapy, 19, 658-664. http://dx.doi.org/10.1179/joc.2007.19.6.658

[11] Muder, R.R., Harris, A.P., Muller, S., Edmond, M., Chow, J.W., Papadakis, K., et al. (1996) Bacteremia Due to Stenotrophomonas (Xanthomonas) Maltophilia: A Prospective, Multicenter Study of 91 Episodes. Clinical Infectious Diseases, 22, 508-512. http://dx.doi.org/10.1093/clinids/22.3.508

[12] Elting, L.S. and Bodey, G.P. (1990) Septicemia Due to Xanthomonas Species and Non-Aeruginosa Pseudomonas Species: Increasing Incidence of Catheter-Related Infections. Medicine, 69, 296-306. http://dx.doi.org/10.1097/00005792-199009000-00003

[13] Paez, J.I.G. and Costa, S.F. (2008) Risk Factors Associated with Mortality of Infections Caused by Stenotrophomonas maltophilia: A Systematic Review. Journal of Hospital Infection, 70, 101-108. 
http://dx.doi.org/10.1016/j.jhin.2008.05.020

[14] Del Toro, M.D., Rodríguez-Baño, J., Herrero, M., Herrero, M., Rivero, A., García-Ordoñez, M.A., et al. (2002) Clinical Epidemiology of Stenotrophomonas maltophilia Colonization and Infection: A Multicenter Study. Medicine, 81, 228-239. http://dx.doi.org/10.1097/00005792-200205000-00006

[15] Carmeli, Y. and Samore, M.H. (1997) Comparison of Treatment with Imipenem vs. Ceftazidime as a Predisposing Factor for Nosocomial Acquisition of Stenotrophomonas maltophilia: A Historical Cohort Study. Clinical Infectious Diseases, 24, 1131-1134. http://dx.doi.org/10.1086/513652

[16] Denton, M. and Kerr, K.G. (1998) Microbiological and Clinical Aspects of Infection Associated with Stenotrophomonas maltophilia. Clinical Microbiology Reviews, 11, 57-80.

[17] Khardori, N., Elting, L., Wong, E., Schable, B. and Bodey, G.P. (1990) Nosocomial Infections Due to Xanthomonas maltophilia (Pseudomonas maltophilia) in Patients with Cancer. Clinical Infectious Diseases, 12, 997-1003. http://dx.doi.org/10.1093/clinids/12.6.997

[18] Araoka, H., Baba, M. and Yoneyama, A. (2010) Risk Factors for Mortality among Patients with Stenotrophomonas maltophilia Bacteremia in Tokyo, Japan, 1996-2009. European Journal of Clinical Microbiology \& Infectious Diseases, 29, 605-608. http://dx.doi.org/10.1007/s10096-010-0882-6

[19] Platsouka, E., Routsi, C., Chalkis, A., Dimitriadou, E., Paniara, O. and Roussos, C. (2002) Stenotrophomonas maltophilia Meningitis, Bacteremia and Respiratory Infection. European Journal of Clinical Microbiology \& Infectious Diseases, 34, 391-392. http://dx.doi.org/10.1080/00365540110080520

[20] Crum, N.F., Utz, G.C. and Wallace, M.R. (2002) Stenotrophomonas maltophilia Endocarditis. Scandinavian Journal of Infectious Diseases, 34, 925-927.

[21] Comité de L’Antibiogramme Société Française de Microbiologie (CASFM). Editions de 2002 à 2011.

[22] Micozzi, A., Venditti, M., Monaco, M., Friedrich, A., Taglietti, F., Santilli, S., et al. (2000) Bacteremia Due to Stenotrophomonas maltophilia in Patients with Hematologic Malignancies. Clinical Infectious Diseases, 31, 705-711. http://dx.doi.org/10.1086/314043

[23] Victor, M.A., Arpi, M., Bruun, B., Jonsson, V. and Hansen, M.M. (1994) Xanthomonas maltophilia Bacteremia in Immunocompromised Hematological Patients. Scandinavian Journal of Infectious Diseases, 26, 163-170. http://dx.doi.org/10.3109/00365549409011780

[24] Elting, L.S., Khardori, N., Bodey, G.P. and Fainstein, V. (1990) Nosocomial Infection Caused by Xanthomonas maltophilia: A Case-Control Study of Predisposing Factors. Infection Control and Hospital Epidemiology, 11, 134-138. http://dx.doi.org/10.2307/30145475

[25] Wang, W.S., Liu, C.P., Lee, C.M. and Huan, F.Y. (2004) Stenotrophomonas maltophilia Bacteremia in Adults: Four Years Experience in a Medical Center in Northern Taiwan. Journal of Microbiology, Immunology and Infection, 37, 359-365.

[26] Cheong, H.S., Lee, J.A., Kang, C.I., Chung, D.R., Peck, K.R., Kim, E.S., et al. (2008) Risk Factors for Mortality and Clinical Implications of Catheterrelated Infections in Patients with Bacteraemia Caused by Stenotrophomonas maltophilia. International Journal of Antimicrobial Agents, 32, 538-547. http://dx.doi.org/10.1016/j.ijantimicag.2008.05.011

[27] Nseir, S., Di Pompeo, C., Brisson, H., Dewavrin, F., Tissier, S., Diarra, M., et al. (2006) Intensive Care Unit-Acquired Stenotrophomonas maltophilia: Incidence, Risk Factors, and Outcome. Critical Care, 10, R143. http://dx.doi.org/10.1186/cc5063

[28] Metan, G. and Uzun, O. (2005) Impact of Initial Antimicrobial Therapy in Patients with Bloodstream Infections Caused by Stenotrophomonas maltophilia. Antimicrobial Agents and Chemotherapy, 49, 3980-3981. http://dx.doi.org/10.1128/AAC.49.9.3980-3981.2005

[29] Ubeda, P., Salavert M., Giner, S., Jarque, I., López-Aldeguer, J., Pérez-Bellés, C. and Gobernado, M. (1998) Bac-teremia Caused by Stenotrophomonas maltophilia: A Clinical-Epidemiological Study and Resistance Profile. Revista espanola de quimioterapia, 11, 205-215.

[30] Villarino, M.E., Stevens, L.E., Schable, B., Mayers, G., Miller, J.M., Burke, J.P., et al. (1992) Risk Factors for Epidemic Xanthomonas maltophilia Infection/Colonization in Intensive Care Unit Patients. Infection Control and Hospital Epidemiology, 13, 201-206. http://dx.doi.org/10.2307/30147098

[31] Ansari, S.R., Hanna, H., Hachem, R., Jiang, Y., Rolston, K. and Raad, I. (2007) Risk Factors for Infections with Multidrug-Resistant Stenotrophomonas maltophilia in Patients with Cancer. Cancer, 109, 2615-2622. http://dx.doi.org/10.1002/cncr.22705

[32] Gopalakrishnan, R., Hawley, H.B., Czachor, J.S., Markert, R.J. and Bernstein, J.M. (1999) Stenotrophomonas maltophilia Infection and Colonization in the Intensive Care Units of Two Community Hospitals. A Study of 143 Patients. Heart \& Lung: The Journal of Acute and Critical Care, 28, 134-141. http://dx.doi.org/10.1053/hl.1999.v28.a96418 
[33] Boktour, M., Hanna, H., Ansari, S., Bahna, B., Hachem, R., Tarrand, J., Rolston, K., Safdar, A. and Raad, I. (2006) Central Venous Catheter and Stenotrophomonas maltophilia Bacteremia in Cancer Patients. Cancer, 106, 1967-1973. http://dx.doi.org/10.1002/cncr.21846

[34] Tsai, W.P., Chen, C.L., Ko, W.C. and Pan, S.C. (2006) Stenotrophomonas maltophilia Bacteremia in Burn Patients. Burns, 32, 155-158. http://dx.doi.org/10.1016/j.burns.2005.08.016

[35] Jang, T.N., Wang, F.D., Wang, L.S., Liu, C.Y. and Liu, I.M. (1992) Xanthomonas maltophilia Bacteremia: An Analysis of 32 Cases. Journal of the Formosan Medical Association, 91, 1170-1176.

[36] Noskin, G.A. and Grohmann, S.M. (1992) Xanthomonas maltophilia Bacteremia: An Analysis of Factors Influencing Outcome. Infectious Diseases in Clinical Practice, 1, 230-236. http://dx.doi.org/10.1097/00019048-199207000-00003

[37] Paez, J.I., Tengan, F.M., Barone, A.A., Levin, A.S. and Costa, S.F. (2008) Factors Associated with Mortality in Patients with Bloodstream Infection and Pneumonia Due to Stenotrophomonas maltophilia. European Journal of Clinical Microbiology \& Infectious Diseases, 27, 901-906. http://dx.doi.org/10.1007/s10096-008-0518-2

[38] Lai, C.H., Wong, W.W., Chin, C., Huang, C.K., Lin, H.H., Chen, W.F., et al. (2006) Central Venous Catheter-Related Stenotrophomonas maltophilia Bacteraemia and Associated Relapsing Bacteraemia in Haematology and Oncology Patients. Clinical Microbiology and Infection, 12, 986-991. http://dx.doi.org/10.1111/j.1469-0691.2006.01511.x

[39] Rodloff, A.C., Goldstein, E.J.C. and Torres, A. (2006) Two Decades of Imipenem Therapy. Journal of Antimicrobial Chemotherapy, 58, 916-929. http://dx.doi.org/10.1093/jac/dkl354

[40] Meyer, E., Schwab, F., Gastmeier, P., Rueden, H., Daschner, F.D. and Jonas, D. (2006) Stenotrophomonas maltophilia and Antibiotic Use in German Intensive Care Units: Data from Project SARI (Surveillance of Antimicrobial Use and Antimicrobial Resistance in German Intensive Care Units. Journal of Hospital Infection, 64, 238-243. http://dx.doi.org/10.1016/j.jhin.2006.07.006

[41] Raffenberg, M., Szymanski, T., Lubasch, A., Erbes, R., Wagner, S., Weist, K., et al. (2001) Infections Caused by Stenotrophomonas maltophilia (SMA) in Intensive Care Patients: A Prospective Case-Control Study. Dtsch Med Wochenschr, 126, 514-518. http://dx.doi.org/10.1055/s-2001-13294

[42] Vartivarian, S.E., Papadakis, K.A. and Anaissie, E.J. (1996) Stenotrophomonas (Xanthomonas) Maltophilia Urinary Tract Infection. A Disease That Is Usually Severe and Complicated. Archives of Internal Medicine, 156, 433-435. http://dx.doi.org/10.1001/archinte.1996.00440040111012

[43] Betriu, C., Sánchez, A., Palau, M.L., Gómez, M. and Picazo, J.J. (2001) Antibiotic Resistance Surveillance of Stenotrophomonas maltophilia, 1993-1999. Journal of Antimicrobial Chemotherapy, 48, 152-154. http://dx.doi.org/10.1093/jac/48.1.152

[44] Safdar, A. and Rolston, K.V. (2007) Stenotrophomonas maltophilia: Changing Spectrum of a Serious Bacterial Pathogen in Patients with Cancer. Clinical Infectious Diseases, 45, 1602-1609. http://dx.doi.org/10.1086/522998

[45] Wu, P.S., Lu, C.Y., Chang, L.Y., Hsueh, P.R., Lee, P.I., Chen, J.M., et al. (2006) Stenotrophomonas maltophilia Bacteremia in Pediatric Patients-A 10-Year Analysis. Journal of Microbiology, Immunology and Infection, 39, $144-149$.

[46] Zelenitsky, S.A., Iacovides, H., Ariano, R.E. and Harding, G.K.M. (2005) Antibiotic Combinations Significantly More Active than Monotherapy in an in Vitro Infection Model of Stenotrophomonas maltophilia. Diagnostic Microbiology and Infectious Disease, 51, 39-43. http://dx.doi.org/10.1016/j.diagmicrobio.2004.09.002

[47] Falagas, M.E., Valkimadi, P.E., Huang, Y.T., Matthaiou, D.K. and Hsueh, P.R. (2008) Therapeutic Options for Stenotrophomonas maltophilia Infections Beyond Co-Trimoxazole: A Systematic Review. Journal of Antimicrobial Chemotherapy, 62, 889-894. http://dx.doi.org/10.1093/jac/dkn301

[48] Valdezate, S., Vindel, A., Loza, E., Baquero, F. and Cantón, R. (2001) Antimicrobial Susceptibilities of Unique Stenotrophomonas maltophilia Clinical Strains. Antimicrobial Agents and Chemotherapy, 45, 1581-1584. http://dx.doi.org/10.1128/AAC.45.5.1581-1584.2001

[49] Gales, A.C., Jones, R.N., Forward, K.R., Linares, J., Sader, H.S. and Verhoef, J. (2001) Emerging Importance of Multi-Drug Resistant Acinetobacter Species and Stenotrophomonas maltophilia as Pathogens in Severely Ill Patients: Geographic Patterns, Epidemiological Features and Trends in the SENTRY Antimicrobial Surveillance Program (1997-1999). Clinical Infectious Diseases, 32, 104-113. http://dx.doi.org/10.1086/320183

[50] Nicodemo, A.C. and Paez, J.I.G. (2007) Antimicrobial Therapy for Stenotrophomonas maltophilia Infections. European Journal of Clinical Microbiology \& Infectious Diseases, 26, 229-237. http://dx.doi.org/10.1007/s10096-007-0279-3

[51] Tsiodras, S., Pittet, D., Carmeli, Y., Eliopoulos, G., Boucher, H. and Harbath, S. (2000) Clinical Implications of Stenotrophomonas maltophilia Resistant to Trimethoprim-Sulfamethoxazole: A Study of 69 Patients at 2 University Hospitals. Scandinavian Journal of Infectious Diseases, 32, 651-656. http://dx.doi.org/10.1080/003655400459577

[52] Gabriel, P.S., Zhou, J., Tabibi, S., Chen, Y., Trauzzi, M. and Saiman, L. (2004) Antimicrobial Susceptibility and Synergy Studies of Stenotrophomonas maltophilia Isolates from Patients with Cystic Fibrosis. Antimicrobial Agents and 
Chemotherapy, 28, 168-171. http://dx.doi.org/10.1128/AAC.48.1.168-171.2004

[53] Betriu, C., Rodríguez-Avial, I., Sánchez, B.A., Gómez, M. and Picazo, J.J. (2002) Comparative in Vitro Activities of Tigecycline (GAR-936) and Other Antimicrobial Agents against Stenotrophomonas maltophilia. Journal of Antimicrobial Chemotherapy, 50, 758-759. http://dx.doi.org/10.1093/jac/dkf196

[54] Betriu, C., Rodriguez-Avial, I., Sánchez, B.A., Gómez, M., Alvarez, J. and Picazo, J.J. (2002) In Vitro Activities of Tigecycline (GAR-936) against Recently Isolated Clinical Bacteria in Spain. Antimicrobial Agents and Chemotherapy, 46, 892-895. http://dx.doi.org/10.1128/AAC.46.3.892-895.2002

[55] Bonfiglio, G., Cascone, C., Azzarelli, C., Cafiso, V., Marchetti, F. and Stefani, S. (2000) Levofloxacin in Vitro Activity and Time-Kill Evaluation of Stenotrophomonas maltophilia Clinical Isolates. Journal of Antimicrobial Chemotherapy, 45, 115-117. http://dx.doi.org/10.1093/jac/45.1.115

[56] Al-Jasser, A.M. (2006) Stenotrophomonas maltophilia Resistant to Trimethoprim-Sulfamethoxazole: An Increasing Problem. Annals of Clinical Microbiology and Antimicrobials, 5, 23. http://dx.doi.org/10.1186/1476-0711-5-23

[57] Brooke, J.S. (2012) Stenotrophomonas maltophilia: An Emerging Global Opportunistic Pathogen. Clinical Microbiology Reviews, 25, 1-41. http://dx.doi.org/10.1128/CMR.00019-11

[58] Liaw, S.J., Teng, L.J., Hsueh, P.R., Ho, S.W. and Luh, K.T. (2002) In Vitro Activities of Antimicrobial Combinations against Clinical Isolates of Stenotrophomonas maltophilia. Journal of the Formosan Medical Association, 101, 495501.

[59] Yeshurun, M., Gafter-Gvili, A., Thaler, M., Keller, N., Nagler, A. and Shimoni, A. (2010) Clinical Characteristics of Stenotrophomonas maltophilia Infection in Hematopoietic Stem Cell Transplantation Recipients: A Single Center Experience. Infection, 38, 211-215. http://dx.doi.org/10.1007/s15010-010-0023-2 